\title{
Multiband Radar Signal Coherent Processing Algorithm for Motion Target
}

\author{
Tingjing Wang, Ying Zhang, Hua Zhao, and Yanxin Zhang \\ College of Electronic Engineering, University of Electronic Science and Technology of China, Chengdu, Sichuan 611731, China \\ Correspondence should be addressed to Ying Zhang; zhying@uestc.edu.cn
}

Received 24 April 2017; Revised 6 June 2017; Accepted 22 June 2017; Published 20 November 2017

Academic Editor: Yumao Wu

Copyright (C) 2017 Tingjing Wang et al. This is an open access article distributed under the Creative Commons Attribution License, which permits unrestricted use, distribution, and reproduction in any medium, provided the original work is properly cited.

\begin{abstract}
In real application, most aerial targets are movable. In this paper, an effective multiple subbands coherent processing method is proposed for moving target. Firstly, an echoed signal model of motion target based on geometrical theory of diffraction is established and the influence of velocity on range profile of the target is analyzed. Secondly, a method based on minimum entropy principle is used to compensate velocity. Then, incoherent factors including a quadratic phase term, a linear phase factor, a fixed factor, and an amplitude difference term are analyzed. Subsequently, efficient methods are applied to estimate other incoherent factors, except that the quadratic term is small enough to be ignored. Finally, the feasibility and performance of the proposed method are investigated through numerical simulation.
\end{abstract}

\section{Introduction}

Multiple radar subband fusion is effective to obtain ultrawide bandwidth so that the range resolution of radar target can be improved. Compared with real ultra-wideband radar, it reduces the manufacture costs and the complexity of system design. Since the received echoes with respect to different radars differ in time delay, amplitude, and so forth, coherent compensation between subbands is essential before multiband fusion [1-5].

In [2], Cuomo et al. obtained the target echoed signal from distributed radars. Then, a nonlinear optimization problem was established to estimate the pole rotation angle and the complex amplitude coefficient. However, the procedure of solving the function was rather complicated. Besides, Wang [5] proposed to use modern spectral estimation methods including modified root-multiple signal classification (MUSIC) algorithm and estimated signal parameters via rotational invariance technique (ESPRIT) to estimate the incoherent factors. However, these methods require overlapped observation frequency bands. In [6,7], all-phase fast Fourier transform (apFFT) spectral analysis was proposed to obtain the phase factor between subbands. It shows good performance when estimating incoherent factors without the prior information of scattering centers numbers. However, the estimation precision of the coherent factor is limited by the sampling frequency. A coherent processing method based on sparse representation was present in [8]; genetic algorithm (GA) was adopted to obtain optimal parameters. GA can simultaneously optimize multiple parameters and solve out superior estimates but it is computationally demanding when parameters increase.

The aforementioned algorithms assumed that the target is static or with low speed. However, most aerial targets tend to be fast moving in real applications [9]. In this paper, in order to take a more practical situation into account, a new subband coherent compensation method is presented. The proposed method consists of the following steps. Firstly, an echoed signal based on geometrical theory of diffraction (GTD) model of constant high speed target is built and the influence of velocity on range profile is analyzed. Then, an effective method using minimum entropy principle is applied to compensate the velocity. Considering the residual of velocity compensation and difference of time delays and amplitudes between subbands, the incoherent factors are analyzed, which are shown to include a quadratic phase term, a linear phase factor, a fixed factor, and an amplitude difference term. Quadratic term is so small which can be ignored. Subsequently, a modified cross-correction method is applied to estimate the linear phase factor. As 
TABLE 1: Scattering structures and the corresponding geometry parameters.

\begin{tabular}{lccccc}
\hline Scattering field & Corner & Edge & Point & Single curved surface & Flat plate \\
\hline$\alpha$ & -1 & -0.5 & 0 & 0.5 \\
\hline
\end{tabular}

for the fixed phase factor, the one-dimension optimization problem is transformed to nonlinear least square fitting problem to improve the estimation precision. Taking amplitude difference into account, golden section method is applied to seek an appropriate amplitude factor. To verify validity of the proposed algorithm, computer simulation is conducted.

The rest of this paper is organized as follows: in Section 2, radar echo model of motion target is established and the influence of the velocity on range resolution is analyzed. Velocity compensation and incoherent parameters estimation are elaborated in Section 3. After that, computer simulation result is shown in Section 4. Finally, discussion and conclusion are drawn in Section 5.

\section{Analysis of Radar Echoed Signal of Motion Target}

Without considering the resonance behavior of a target to wideband systems, it is generally believed that the target scattering characteristics of two radars are approximately in agreement. The frequency response of echoed signal is described by the following GTD model:

$$
S_{R i}\left(f_{i}\right)=S_{i}\left(f_{i}\right) \sum_{m=1}^{M} A_{m i}\left(j \frac{f_{i}}{f_{c i}}\right)^{\alpha_{m}} \exp \left(-j \frac{4 \pi R_{m i} f_{i}}{c}\right)
$$

where $S_{i}(f)$ denotes the frequency spectrum of the transmitted signals of the $i$ th radar, $i=1,2 . f_{i}=f_{0}+$ $n_{i} \Delta f_{i}$, where $f_{0}$ represents the initial frequency, $n_{i}$ is the $n_{i}$ th frequency point, and the frequency step is denoted by $\Delta f_{i}$. $f_{c i}$ denotes the carrier frequency. $A_{m i}$ and $R_{m i}$ denote the complex amplitude and range of the $m$ th scattering center for the $i$ th radar, respectively. Geometry parameter of the $m$ th scattering center is denoted by $\alpha_{m}$. In particular, Table 1 shows the scattering structures and the corresponding geometry parameters. $c$ is the light speed.

Then, (1) can be rewritten as

$$
\begin{aligned}
S_{R i}\left(f_{i}\right) & =\sum_{m=1}^{M} A_{m i}\left(j \frac{f_{i}}{f_{c}}\right)^{\alpha_{m}} S_{i}^{\prime}\left(f_{i}\right), \\
S_{i}^{\prime}\left(f_{i}\right) & =S_{i}\left(f_{i}\right) \exp \left(-j \frac{4 \pi R_{m i} f_{i}}{c}\right) ;
\end{aligned}
$$

(4) is transformed to time domain, namely,

$$
S_{i}^{\prime}(t)=S_{i}\left(t-\frac{2 R_{m i}}{c}\right)
$$

When radar target is static or with low speed, the distance between target and radar can be considered invariant within the pulse duration. However, when target is fast moving, this assumption does not hold. Supposing that $v_{m}$ is the radial velocity of the $m$ th scattering centers, the instantaneous distance $\widetilde{R}_{m i}$ at time $t$ is given by $\widetilde{R}_{m i}=R_{m i}+v_{m} t$.

Substituting (4) into (3) yields

$$
\begin{aligned}
& S_{i}^{\prime}(t)=\operatorname{rect}\left(\frac{a_{m} t-b_{m i}}{T_{i}}\right) \\
& \cdot \exp \left(\left[j 2 \pi\left(f_{c i}\left(a_{m} t-b_{m i}\right)+\frac{1}{2} K_{i}\left(a_{m} t-b_{m i}\right)^{2}\right)\right]\right), \\
& a_{m}=1-\frac{2 v_{m}}{c} \\
& b_{m i}=\frac{2 R_{m i}}{c}
\end{aligned}
$$

therefore,

$$
S_{R i}\left(f_{i}\right)=\sum_{m=1}^{M} A_{m i}\left(j \frac{f_{i}}{f_{c}}\right)^{\alpha_{m}} \cdot F\left(S_{i}^{\prime}(t)\right) .
$$

$F(\cdot)$ is Fourier transform. $S_{R i}\left(f_{i}\right)$ can be further induced by stationary phase principle and let $f_{i}=f_{i}+f_{c i}$;

$$
\begin{aligned}
S_{R i}\left(f_{i}\right)= & \sum_{m=1}^{M} \bar{A}_{m i}\left(j \frac{f_{i}+f_{c i}}{f_{c i}}\right)^{\alpha_{m}} \\
& \cdot \operatorname{rect}\left(\frac{f_{i}+\left(2 v_{m} / c\right) f_{c i}}{a_{m} B_{i}}\right) \exp \left(j \Phi_{m i}\left(f_{i}\right)\right),
\end{aligned}
$$

where

$$
\begin{aligned}
\Phi_{m i}(f)= & -\frac{\pi\left(f_{i}+\left(2 v_{m} / c\right) f_{c i}\right)^{2}}{K_{i} a_{m}^{2}}-\frac{2 \pi b_{m i}}{a_{m}}\left(f_{i}+f_{c i}\right) \\
& +\frac{\pi}{4},
\end{aligned}
$$

and $\bar{A}_{m i}=\left(1 / a_{m} \sqrt{K_{i}}\right) A_{m i}$, and the pulse widths and bandwidth are denoted by $T_{i}$ and $B_{i}$, respectively. $K_{i}=B_{i} / T_{i}$ is the frequency modulated rate. Process the echoed signal with matching filter,

$$
M_{R i}\left(f_{i}\right)=S_{R i}\left(f_{i}\right) \cdot S_{\text {ref } \_i^{*}}\left(f_{i}\right)
$$

and $S_{\mathrm{ref} i}\left(f_{i}\right)$ is the spectrum of conference signal $S_{\text {ref } i}(t)$.

$$
S_{\text {ref } \_i}(t)=\exp \left(\left[j \pi K_{i} t^{2}\right]\right) .
$$




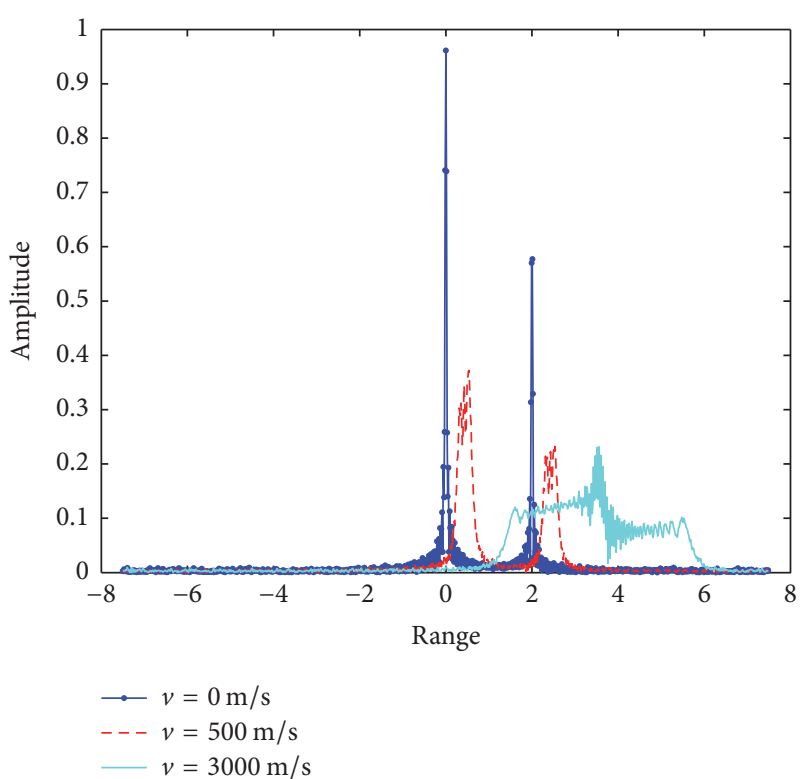

FIGURE 1: The influence of varied target velocity on range profile.

Then the echoed signal after matching filter can be expressed as

$$
\begin{aligned}
& M_{R i}\left(f_{i}\right)=\sum_{m=1}^{M} \widetilde{A}_{m i} \operatorname{rect}\left(\frac{f_{i}+\left(2 v_{m} / c\right) f_{c i}}{\left(1-2 v_{m} / c\right) B_{i}}\right)\left(j \frac{f_{i}+f_{c i}}{f_{c i}}\right)^{\alpha_{m}} \exp \left(j \Phi_{m i}^{\prime}\left(f_{i}\right)\right), \\
& \Phi_{m i}^{\prime}\left(f_{i}\right)=-\frac{4 \pi v_{m}\left(c-v_{m}\right)}{K_{i}\left(c-2 v_{m}\right)^{2}} f_{i}^{2}-\frac{4 \pi v_{m} c f_{c i}}{K_{i}\left(c-2 v_{m}\right)^{2}} f_{i} \\
& \quad-\frac{4 \pi v_{m}^{2} f_{c i}^{2}}{K_{i}\left(c-2 v_{m}\right)^{2}}-\frac{4 \pi R_{m i}}{c-2 v_{m}}\left(f_{i}+f_{c i}\right) \\
& \approx-\frac{4 \pi v_{m}}{K_{i}\left(c-2 v_{m}\right)} f_{i}^{2}-\frac{4 \pi v_{m} c f_{c i}}{K_{i}\left(c-2 v_{m}\right)^{2}} f_{i} \\
& -\frac{4 \pi v_{m}^{2} f_{c i}^{2}}{K_{i}\left(c-2 v_{m}\right)^{2}}-\frac{4 \pi R_{m i}}{c-2 v_{m}}\left(f_{i}+f_{c i}\right),
\end{aligned}
$$

where $v_{m} \ll c$ and $\widetilde{A}_{m i}=\left(1 / a_{m} K_{i}\right) A_{m i}$. From (12), when the target moves, the change of scale and location of the range profile occur. When the speed is low or frequency modulated rate is high, the second-order term in (12) can be ignored. On the contrary, serious influence may be triggered by high speed or low frequency modulated rate. Processing the echoed signal using inverse fast Fourier transform (IFFT) to obtain range profile, the quadratic term in (12) may lead to distortion of range profile of target, which is shown as reduction of peak values and broadening of waveform. Besides, the second term in (12) may give rise to the shift of range profile. Figure 1 shows the influence of varied target velocity on range profile. It is observed that the range profile deteriorates as the increase of target velocity.

\section{Coherent Processing Algorithm with Velocity Compensation}

The procedure of multiband coherent processing is presented in Figure 2. The steps include the following:

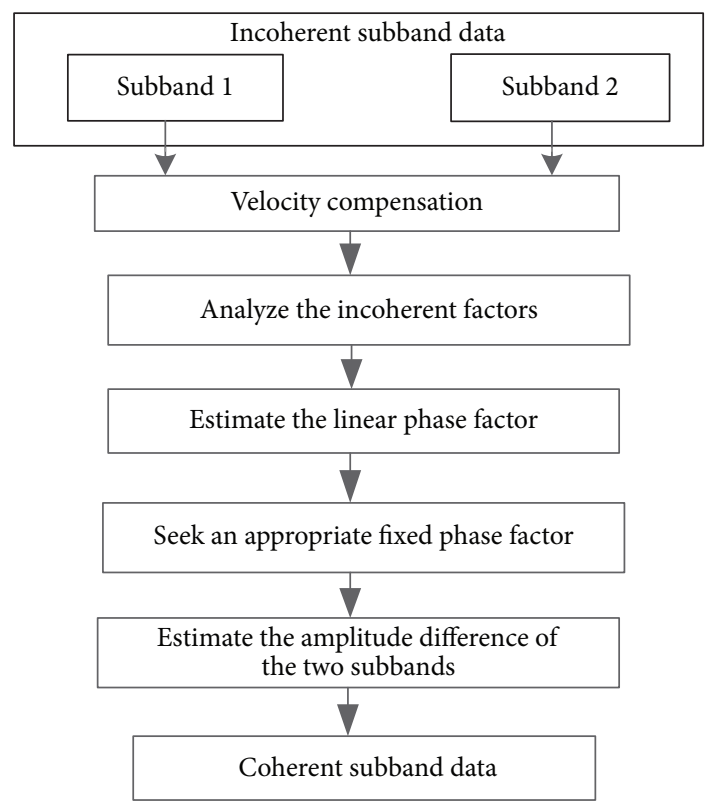

Figure 2: The flow of multiple subband coherent processing.

(1) Estimate target moving speed and apply velocity compensation to subband signals.

(2) Analyze the incoherent factors and establish a nonlinear optimization function to estimate the factors.

(3) Estimate the linear phase term by cross-correlation method.

(4) Solve a nonlinear least square fitting problem to seek the fixed phase factor.

(5) Estimate the amplitude difference between the subbands by golden section method.

(6) Coherent recovery using the estimated incoherent factors.

3.1. Velocity Compensation and Phase Residual Analysis. From the aforementioned analysis, it is pretty critical to eliminate or decrease the influence of target velocity before coherent processing.

Assume that $\varphi_{i}\left(f_{i}\right)$ is the compensatory phase which includes estimation velocity. $y_{i}\left(f_{i}\right)$ represents $M_{R i}\left(f_{i}\right)$ which is compensated by $\varphi_{i}\left(f_{i}\right)$. The one-dimension range profile of $y_{i}\left(f_{i}\right)$ is $Y_{i}=\left\{Y_{i}(h) \mid h=1,2, \ldots, n\right\}$, and the amplitude distribution of sampling point can be computed as

$$
p_{h i}=\frac{\left|Y_{i}(h)\right|^{2}}{\sum_{l=1}^{n}\left|Y_{i}(l)\right|^{2}} .
$$

Using $p_{h}$ to computing the entropy yields

$$
H_{i}=-\sum_{h=1}^{n} p_{h i} \ln p_{h i}
$$


Then, the required velocity compensation phase $\widehat{\varphi}_{i}$ can be obtained by minimizing

$$
\widehat{\varphi}_{i}=\underset{\varphi_{i}}{\arg \min } H
$$

Traversing method is utilized to estimate the velocity [10]. To be specific, search step is set up within the range of certain velocity and entropy of range profile is resolved by compensatory velocity. When $H$ reaches its minimum, the corresponding $\widetilde{V}$ is the most appropriate compensatory velocity. In the view of the velocity values of different scattering centers which are almost identical, $v_{m}$ can be replaced by the uniform estimated velocity $\widetilde{V}$. The echoed signal is then compensated via

$$
y_{i}\left(f_{i}\right)=M_{R i}\left(f_{i}\right) * \exp \left(j \widehat{\varphi}_{i}\left(f_{i}\right)\right),
$$

where

$$
\begin{aligned}
\widehat{\varphi}_{i}\left(f_{i}\right)= & \frac{4 \pi \widetilde{V}}{K_{i}(c-2 \widetilde{V})} f_{i}^{2}+\frac{4 \pi \widetilde{V} c f_{c i}}{K_{i}(c-2 \widetilde{V})^{2}} f_{i} \\
& +\frac{4 \pi \widetilde{V}^{2} f_{c i}^{2}}{K_{i}(c-2 \widetilde{V})^{2}}, \quad i=1,2 .
\end{aligned}
$$

On the assumption that $\left\{y_{1}\left(f_{1 n}\right), n=0, \ldots, N_{1}-1\right\}$ and $\left\{y_{2}\left(f_{2 n}\right), n=N-N_{2}, \ldots, N-1\right\}$ are the preprocessed echoed signals of two radars. The frequency ranges are within $\left[f_{0}, f_{N_{1}-1}\right]$ and $\left[f_{N-N_{2}}, f_{N-1}\right]$. Because the sampling frequency of the subbands may be different, uniform resampling [11] is applied to subband data so that the sampling rate of all subbands is identical. Meanwhile, as shown in Figure 3, subbands are extrapolated, where $\bar{y}_{1}(f)$ and $\bar{y}_{2}(f)$ represent the extended subband data. $f=f_{0}+n \Delta f, n=1, \ldots, N$. $\Delta f=\min \left\{\Delta f_{1}, \Delta f_{2}\right\}$ denotes frequency step of subbands after uniform resampling.

Take $\bar{y}_{1}(f)$ as the reference signal. Assuming that $v \ll$ $c$ and taking the time delay and the initial phase value difference between two radar echoed signals into account, after velocity compensation and simplification, the phase of radar echoed signals become

$$
\begin{aligned}
\widetilde{\Phi}_{1}(f) \approx & -\frac{4 \pi \Delta v c}{K_{1}(c-2 v)(c-2 \widetilde{V})} f^{2} \\
& +\frac{4 \pi \Delta v c f_{c 1}}{K_{1}(c-2 v)(c-2 \widetilde{V})} f-\frac{4 \pi R_{m 1}}{c-2 v} f, \\
\widetilde{\Phi}_{2}(f) \approx- & \frac{4 \pi \Delta v c}{K_{2}(c-2 v)(c-2 \widetilde{V})} f^{2} \\
& +\frac{4 \pi \Delta v c f_{c 2}}{K_{2}(c-2 v)(c-2 \widetilde{V})} f-\frac{4 \pi R_{m 1}}{c-2 v} f \\
& +\alpha n+\beta,
\end{aligned}
$$

where $\Delta \widehat{v}=v-\widetilde{V}$ is velocity estimation error, $\alpha$ denotes the linear phase term caused by time delay difference between the

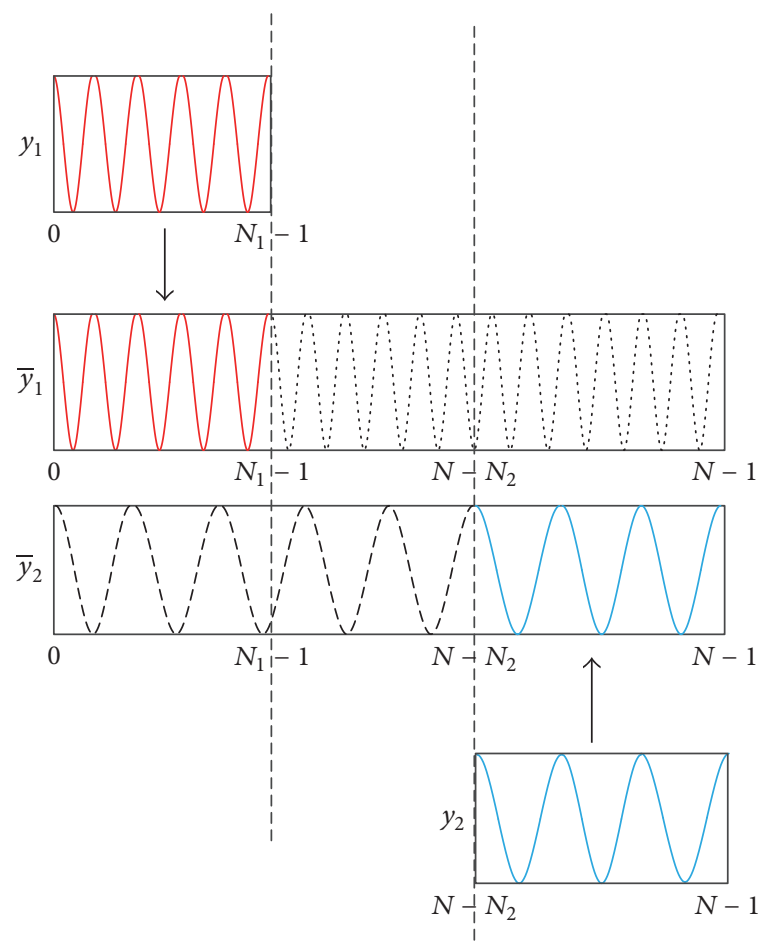

FIGURE 3: Extended subband data for estimation of incoherent factors.

target and the different radars, and $\beta$ denotes the fixed phase difference between subbands. The phase difference between subbands is computed as

$$
\begin{aligned}
\delta(f)= & \widetilde{\Phi}_{1}(f)-\widetilde{\Phi}_{2}(f) \\
\approx & -\left(\frac{1}{K_{1}}-\frac{1}{K_{2}}\right) \frac{4 \pi \Delta v c}{(c-2 v)(c-2 \widetilde{V})} f^{2} \\
& +\left(\left(\frac{f_{c 1}}{K_{1}}-\frac{f_{c 2}}{K_{2}}\right) \frac{4 \pi \Delta v c}{(c-2 v)(c-2 \widetilde{V})}\right) f-\alpha n \\
& -\beta .
\end{aligned}
$$

Discretization processing yields

$\delta(n)$

$$
\begin{aligned}
\approx & -\left(\frac{1}{K_{1}}-\frac{1}{K_{2}}\right) \frac{4 \pi \Delta v \Delta f^{2}}{c} n^{2} \\
& -\left(\left(\frac{1}{K_{1}}-\frac{1}{K_{2}}\right) 2 f_{0}-\left(\frac{f_{c 1}}{K_{1}}-\frac{f_{c 2}}{K_{2}}\right)\right) \frac{4 \pi \Delta v \Delta f}{c} n \\
& -\alpha n \\
& -\left(\left(\frac{1}{K_{1}}-\frac{1}{K_{2}}\right) f_{0}-\left(\frac{f_{c 1}}{K_{1}}-\frac{f_{c 2}}{K_{2}}\right)\right) \frac{4 \pi \Delta v}{c} f_{0} \\
& -\beta .
\end{aligned}
$$

It is observed from (20) that the first, second, and fourth items are the incoherence caused by the residual of velocity 
compensation. The first item of (20) is the quadratic term, which is usually ignored in most literatures. Assuming that $\Delta v=1000 \mathrm{~m} / \mathrm{s}$ and $\Delta f=20 \mathrm{MHZ}$, the bandwidths of two subbands are $1 \mathrm{GHZ}$ and $0.8 \mathrm{GHZ}$ and the pulse widths are $512 \mu \mathrm{s}$. From the formula $-\left(1 / K_{1}-1 / K_{2}\right)\left(4 \pi \Delta v \Delta f^{2} / c\right) n^{2}$, the value of quadratic term is about $0.002145 \mathrm{rad}$. It is obviously small even if the velocity estimation error is large. Therefore, it can be ignored in practical application. A new linear phase factor is composed of second item and initial linear phase factor, which leads to the shift of one-dimension range profile of multiple subbands. The remaining part refers to a new fixed phase, which includes residual term and initial fixed phase factor. It can be concluded that the residual of velocity compensation, as well as time delay, initial phase, and amplitude difference between subbands give rise to the incoherence of the echoed subband signals. It is necessary to estimate the incoherent factors and preprocess the subbands to ensure subband data mutually coherent before subband fusion.

3.2. Incoherent Factors Estimation. In [2], Cuomo et al. proposed that the incoherent factors between different echoed signal spectra can be expressed as linear phase item and fixed phase item when the targets are static or with lower speed. According to (20), besides the linear factor and the fixed factor, amplitude difference and the quadratic term of frequency should also be considered. The following cost function is defined:

$$
J_{1}(\theta, \varphi, \lambda, \rho)=\sum_{n=0}^{N-1}\left|\rho \bar{y}_{1}(n) e^{j\left(\theta n^{2}+\varphi n+\lambda\right)}-\bar{y}_{2}(n)\right|^{2},
$$

where

$$
\begin{aligned}
\theta= & -\left(\frac{1}{K_{1}}-\frac{1}{K_{2}}\right) \frac{4 \pi \Delta v \Delta f^{2}}{c}, \\
\varphi= & -\left(\left(\frac{1}{K_{1}}-\frac{1}{K_{2}}\right) 2 f_{0}-\left(\frac{f_{c 1}}{K_{1}}-\frac{f_{c 2}}{K_{2}}\right)\right) \frac{4 \pi \Delta v \Delta f}{c} \\
& -\alpha, \\
\lambda= & -\left(\left(\frac{1}{K_{1}}-\frac{1}{K_{2}}\right) f_{0}-\left(\frac{f_{c 1}}{K_{1}}-\frac{f_{c 2}}{K_{2}}\right)\right) \frac{4 \pi \Delta v}{c} f_{0} \\
& -\beta .
\end{aligned}
$$

In (21a), $\rho$ is the amplitude difference between the two subbands, $\theta$ denotes the quadratic phase term, and $\varphi$ and $\lambda$ denote new linear phase and fixed phase, respectively.

$J_{1}(\theta, \varphi, \lambda, \rho)$ should be minimized to obtain the optimal estimation of $\theta, \varphi, \lambda$, and $\rho$. It is straightforward to see that (9) is nonlinear and nonconvex. Standard nonlinear optimization algorithms such as Newton method [12] tend to work out local minimum and genetic algorithm [13] can be used to seek a global optimal solution but the burden of calculation is heavy. From prior analysis in Section 3.1, it is deduced that the quadratic term $\theta$ is usually so small, and the value is approximated to zero. Correlation method is applied to deal with the linear phase factor. On the assumption that $R(n)$ is the correlation function of $\bar{y}_{1}(n), \bar{y}_{2}(n)$,

$$
R(n)=\bar{y}_{1}(n) \bar{y}_{2}^{*}(n)
$$

where $(\cdot)^{*}$ is the conjugate operator.

The modeling error is ignored:

$$
\bar{y}_{2}(n) \approx \rho \bar{y}_{1}(n) e^{j(\varphi n+\lambda)} .
$$

We obtained the cross-correlation of range profiles $r(k)$ from $R(n)$ :

$$
\begin{aligned}
r(k) & =\frac{1}{N_{\mathrm{FFT}}} \sum_{n=0}^{N_{\mathrm{FFT}}-1} R(n) e^{j\left(2 \pi / N_{\mathrm{FFT}}\right) n k} \\
& =\frac{1}{N_{\mathrm{FFT}}} \sum_{m=0}^{N_{\mathrm{FFT}}-1} \bar{y}_{1}(n) \bar{y}_{2}^{*}(n) e^{j\left(2 \pi / N_{\mathrm{FFT}}\right) n k} \\
& \approx \frac{\rho e^{-j \lambda}}{N_{\mathrm{FFT}}} \sum_{m=0}^{N-1}\left|\bar{y}_{1}(n)\right|^{2} e^{j\left(2 \pi / N_{\mathrm{FFT}}\right) n(k-(N / 2 \pi) \varphi)},
\end{aligned}
$$

where $N_{\text {FFT }}$ is the number of points in IFFT and $N_{\text {FFT }} \gg N$. Zero-padding is used to expand the length of $R(n)$ to $N_{\mathrm{FFT}}$. When $k=(N / 2 \pi) \varphi, r(k)$ reaches its maximum. Denote this $k$ as $k_{\text {max }}$, and the linear phase is computed as

$$
\widehat{\varphi}=\frac{2 \pi k_{\max }}{N_{\mathrm{FFT}}} .
$$

$\bar{y}_{1}(n)$ is compensated by the linear phase factor in $(25)$; that is,

$$
\bar{y}_{1}^{\prime}(n)=\bar{y}_{1}(n) \cdot \exp (j \widetilde{\varphi} n) .
$$

A new coherent function is defined to estimate the fixed phase factor:

$$
J_{2}(\lambda)=\sum_{n=0}^{N-1}\left|\frac{\bar{y}_{1}^{\prime}(n) e^{j \lambda}}{\left|\bar{y}_{1}^{\prime}(n)\right|}-\frac{\bar{y}_{2}(n)}{\left|\bar{y}_{2}(n)\right|}\right|^{2} .
$$

$J_{2}(\lambda)$ may not be a unimodal function in $[-\pi, \pi]$; in order to gain better coherence, the nonlinear least square problem can be computed by means of MATLAB subroutine lsqcurvefit to find the most appropriate $\hat{\lambda}$.

Substitute $\widehat{\varphi}$ and $\hat{\lambda}$ into the primary cost function (21a), the constant $\hat{\rho}$ can be worked out by golden section method. Our aim is to minimize the function $J_{1}(\rho)$ within the interval $[a, b]$, and the two points $\rho_{1}, \rho_{2}$ are calculated by golden section scale as follows:

$$
\begin{aligned}
& \rho_{1}=a+0.382 *(b-a), \\
& \rho_{2}=a+0.618 *(b-a) .
\end{aligned}
$$

The search interval is chosen as $\left[a, \rho_{2}\right]$, if $J_{1}\left(\rho_{1}\right)<J_{1}\left(\rho_{2}\right)$. Otherwise, the interval is $\left[\rho_{1}, b\right]$. Repeat (28) by new points; in this way, search interval is shrinking, and the point is approximated to the minimum $\hat{\rho}$. 


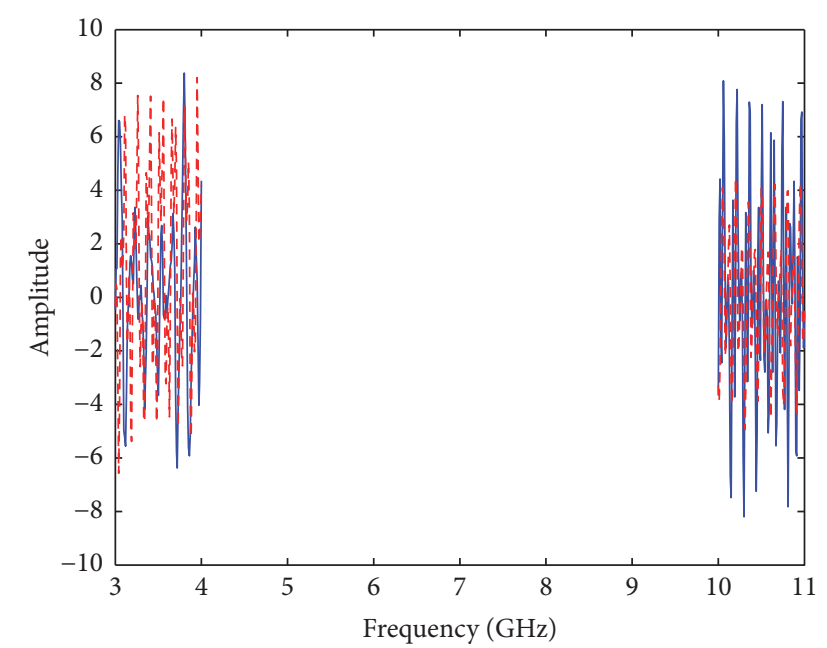

Figure 4: Two subband data sets (blue solid lines denote the real coherent subbands. Red dash line denotes the measured incoherent subbands in the condition that target is moving).

The lower subband signal data is rewritten as follows:

$$
\tilde{y}_{1}(n)=\widehat{\rho} y_{1}(n) e^{j(\hat{\varphi} n+\widehat{\lambda})}, \quad n=1, \ldots, N_{1}-1 .
$$

Now the lower frequency band data in $\left[f_{0}, f_{N_{1}-1}\right]$ is coherent to the upper one. The aforementioned process assumes implicitly that the upper sideband data is more pristine than the lower subband data. However, in most practical application, the choice of benchmark subband data should be made based on sensor hardware or data equality evaluations. Otherwise, the data may be fused but degraded if the data is from lower quality sensor.

\section{Computer Simulation Result}

In this section, the performance and feasibility of the proposed method are verified via numerical simulations. LFM signals are transmitted by two radars, carrier frequencies $f_{c 1}, f_{c 2}$ are $3.5 \mathrm{GHZ}$ and $10.5 \mathrm{GHZ}$, bandwidths $B_{1}, B_{2}$ are $1 \mathrm{GHZ}$ and $0.8 \mathrm{GHZ}$, and both the pulse durations are $100 \mu \mathrm{s}$. Supposing there are four scattering centers of target, the range between radar and scattering centers is $200 \mathrm{Km}$. The echo amplitude of scattering centers is $1,2,3$, and 1 . Geometry parameters of the scattering centers are $0.5,1,0$, and -1 . In particular, velocities are all $3000 \mathrm{~m} / \mathrm{s}$. In order to validate the effectiveness of velocity compensation, set lower subband signal as an example. Simulate the effect of mutual incoherence between the subbands; the signal sampled in the lower subband has been attached to a linear phase factor $\alpha=-\pi / 9$, a fixed phase factor $\beta=-\pi / 4$, and a constant $\rho=1.5$.

It is observed from Figure 4 that red dash lines are not consistent with blue solid lines, because target velocity makes the original subbands shift on frequency spectra. As shown in Figure 5, it is easily concluded that process incoherent subband data directly without velocity compensation leads to mismatching between real lower subband and the measured

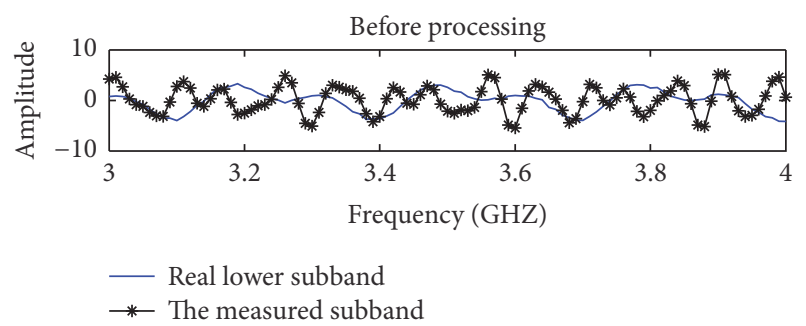

(a)

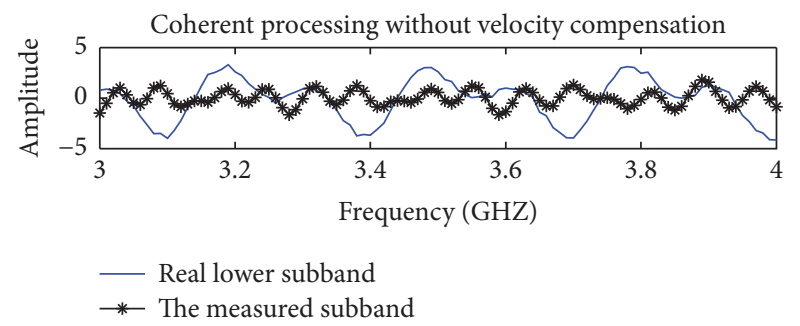

(b)

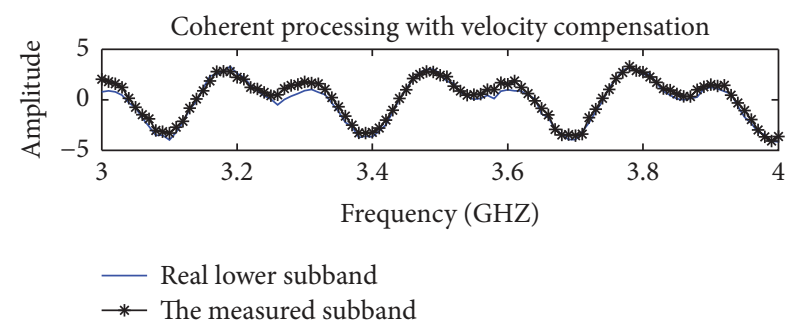

(c)

Figure 5: Comparison between real lower subband and the measured subband ((a) shows the two subbands before processing, (b) shows velocity compensation is not used to process the measured subband, and (c) shows velocity compensation is used to process the measured subband).

subband. Besides, applying velocity compensation method on the measured subband, it matches well with real lower subband after coherent processing.

Furthermore, to test the antinoise performance of the proposed method, zero mean white noise is added to the original signal. After velocity compensation, the pole method proposed in [14] and apFFT method [8] are also applied to process two subband data sets.

Root mean square error (RMSE) is defined as RMSE = $\sqrt{\left(1 / M_{\mathrm{iter}}\right) \sum_{i=1}^{M_{\mathrm{iter}}}\left|\widehat{S}_{i}-s\right|^{2}}$, where $\widehat{S}_{i}$ is estimate result and $s$ is real value. RMSE between real incoherent factors and estimated parameters against the value of signal to noise ratio (SNR) under 100 runs of Monte Carlo simulations is shown in Figure 6. As the figure shows, when estimating the linear phase factors, compared with pole method and apFFT method, our method shows preferable performance when SNR is less than $10 \mathrm{~dB}$, and the accuracy of our method is the most outstanding among all methods. With the increase of SNR, the poled method performs better than other methods. As for the fixed phase factor estimation, RMSE curves evaluated by pole method and apFFT method are approximated, and our method outperforms 


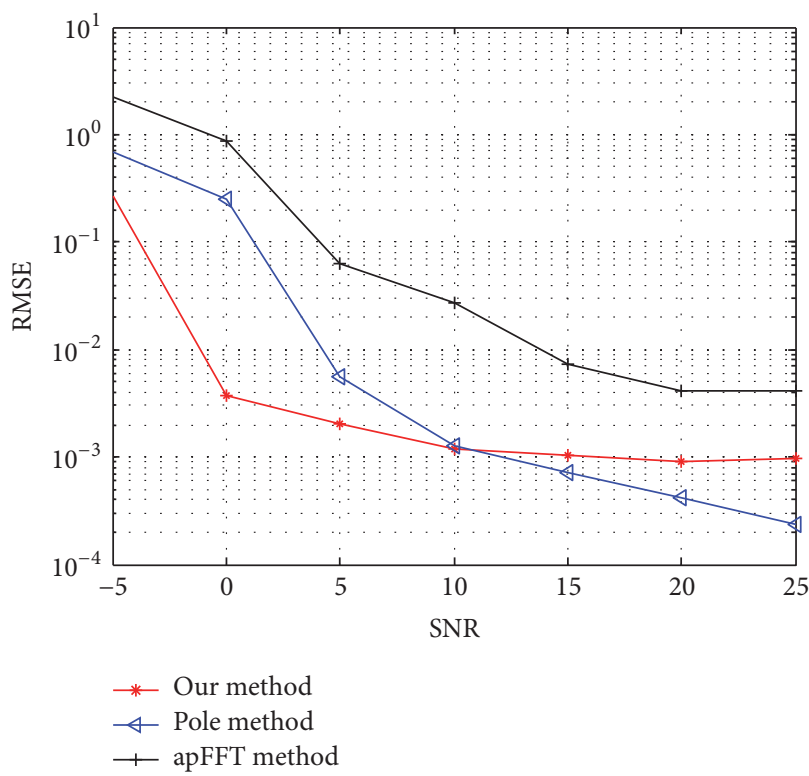

(a) Linear phase

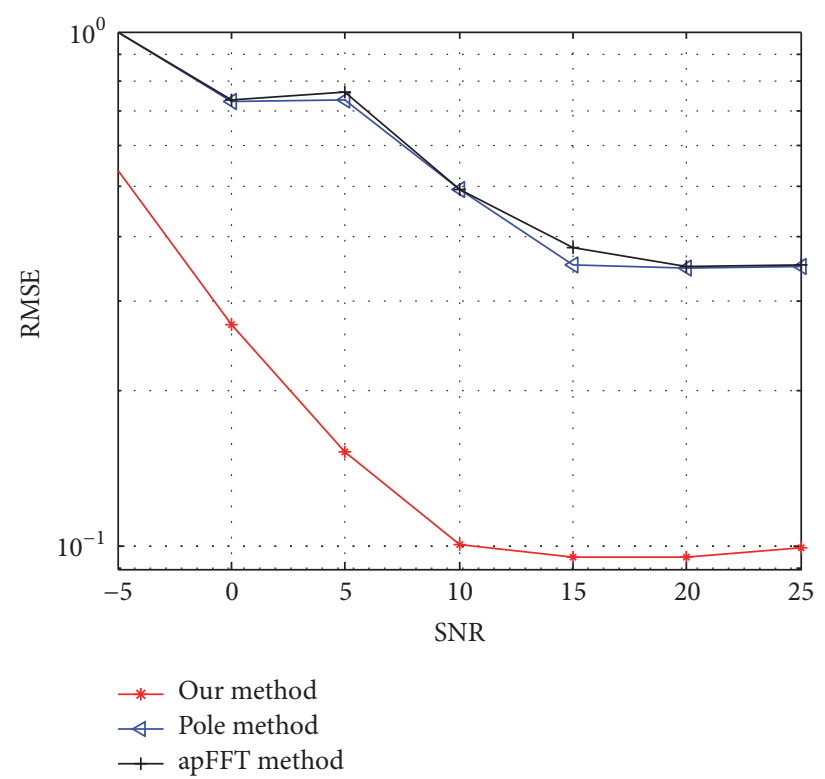

(b) Fixed phase

Figure 6: Average RMSE comparison at different SNR.

the other methods. In conclusion, our method has better antinoise property and improves the incoherent factors estimate precision essentially; also it is more robust than other methods.

\section{Conclusion}

In this paper, a multiple subband coherent processing method based on motion target is proposed. This algorithm firstly introduces an effective velocity compensation approach based on minimum entropy to decrease the effects on range profile of the target. Then, a modified correlation method and a nonlinear least square fitting algorithm are applied to estimate the phase difference between the various subbands. Besides, amplitude error is considered, and golden section algorithm is directly used to search the error. Compared with existing coherent processing methods, the proposed method is able to deal with more practical problem. Simulation results show the robustness in the presence of noise.

\section{Conflicts of Interest}

The authors declare that they have no conflicts of interest.

\section{Acknowledgments}

This paper is supported by a grant from Open Foundation of Key Laboratory of Space Target Measurement, National Science Foundation of China (no. 61671121), Doctoral Fund of Ministry of Education of China (no. 2016M602673), and Doctoral Fund of Jiangxi Province (no. 2016KY15).

\section{References}

[1] S. L. Borison, S. B. Bowling, and K. M. Cuomo, "Superresolution methods for wideband radar," The Lincoln Laboratory Journal, vol. 5, no. 3, pp. 441-461, 1992.

[2] K. Cuomo, J. Pion, and J. Mayhan, "Ultrawide-band coherent processing," IEEE Transactions on Antennas and Propagation, vol. 47, no. 6, pp. 1094-1107, 1999.

[3] L. D. Vann, K. M. Cuomo, J. E. Piou, and J. T. Mayhan, "Multisensor fusion processing for enhanced radar imaging Tech. Rep. TR-1056," Tech. Rep., Lincoln Laboratory, Massachusetts Inst. Technology, Lexington, MA, USA, 2000.

[4] K. Suwa and M. Iwamoto, "A bandwidth extrapolation technique of polarimetric radar data and a recursive method of polarimetric linear prediction coefficient estimation," in Proceedings of the 2003 IGARSS: Learning From Earth's Shapes and Colours, pp. 4329-4331, Toulouse, France, July 2003.

[5] C. Wang, Research on radar signal level fusion imaging techniques [Ph.D. thesis], National University of Defense Technology, Changsha, China, 2006.

[6] J. Tian, J. Sun, G. Wang, Y. Wang, and W. Tan, "Multiband radar signal coherent fusion processing with IAA and apFFT," IEEE Signal Processing Letters, vol. 20, no. 5, pp. 463-466, 2013.

[7] Y. Zou, X. Gao, X. Li, and Y. Liu, "Multiband radar signals coherent compensation with sparse representation," in Proceedings of the 8th International Congress on Image and Signal Processing, CISP 2015, pp. 1085-1089, Shenyang, China, October 2015.

[8] H. H. Zhang and R. S. Chen, "Coherent processing and superresolution technique of multi-band radar data based on fast sparse Bayesian learning algorithm," Institute of Electrical and Electronics Engineers. Transactions on Antennas and Propagation, vol. 62, no. 12, pp. 6217-6227, 2014.

[9] M. Xing, R. Wu, and Z. Bao, "High resolution ISAR imaging of high speed moving targets," Radar, Sonar and Navigation, IEE Proceedings, vol. 152, no. 2, pp. 58-67, 2005. 
[10] J. Yang, Q. L. Bao, and Z. P. Chen, “The implemental methods of high velocity compensation in isar imaging based on entropy minimization," Signal Processing, vol. 25, no. 12, pp. 1861-1866, 2007.

[11] Y. Zou, X. Z. Gao, and X. Li, "High precision coherent compensation for multiband radar data at low SNR," IEEE Signal Processing Letters, vol. 37, no. 1, pp. 48-54, Jan 2015.

[12] S. Boyd and L. Vandenberghe, Convex Optimization, Cambridge University Press, 2004.

[13] Z. Yu, Y. Chen, Z.-Q. Sun, and Y.-B. Lu, "The super-resolution range imaging based on multiband wideband signal fusion," in Proceedings of the 2009 Asia-Pacific Conference on Synthetic Aperture Radar, APSAR 2009, pp. 160-164, chn, October 2009.

[14] B. Tian, Z. Chen, and S. Xu, "Sparse subband fusion imaging based on parameter estimation of geometrical theory of diffraction model," IET Radar, Sonar and Navigation, vol. 8, no. 4, pp. 318-326, 2014. 


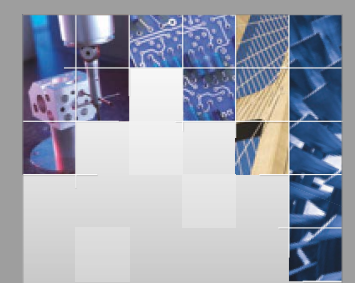

\section{Enfincering}
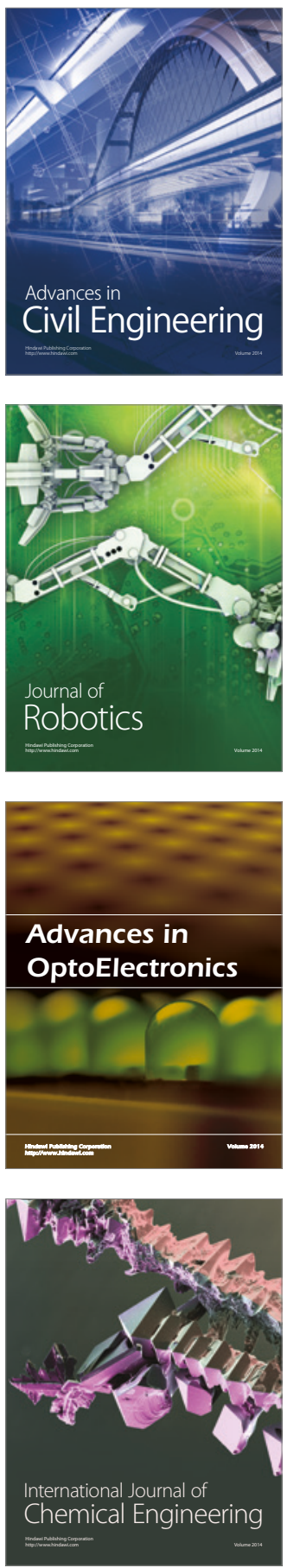

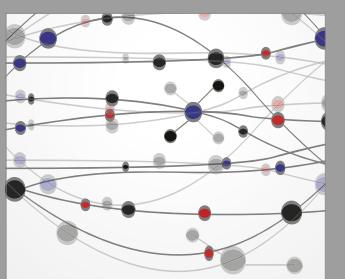

The Scientific World Journal

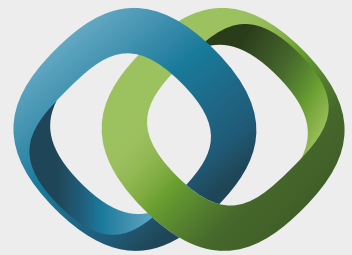

\section{Hindawi}

Submit your manuscripts at

https://www.hindawi.com
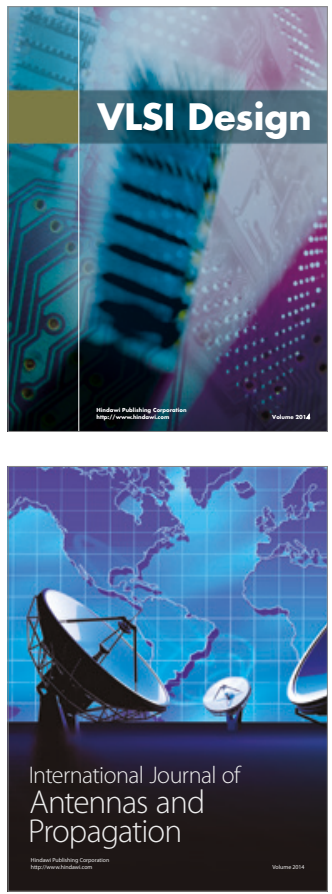

\section{Rotating}

Machinery
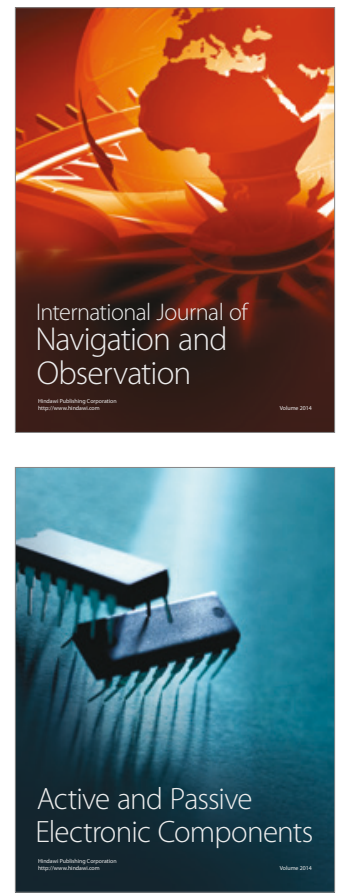
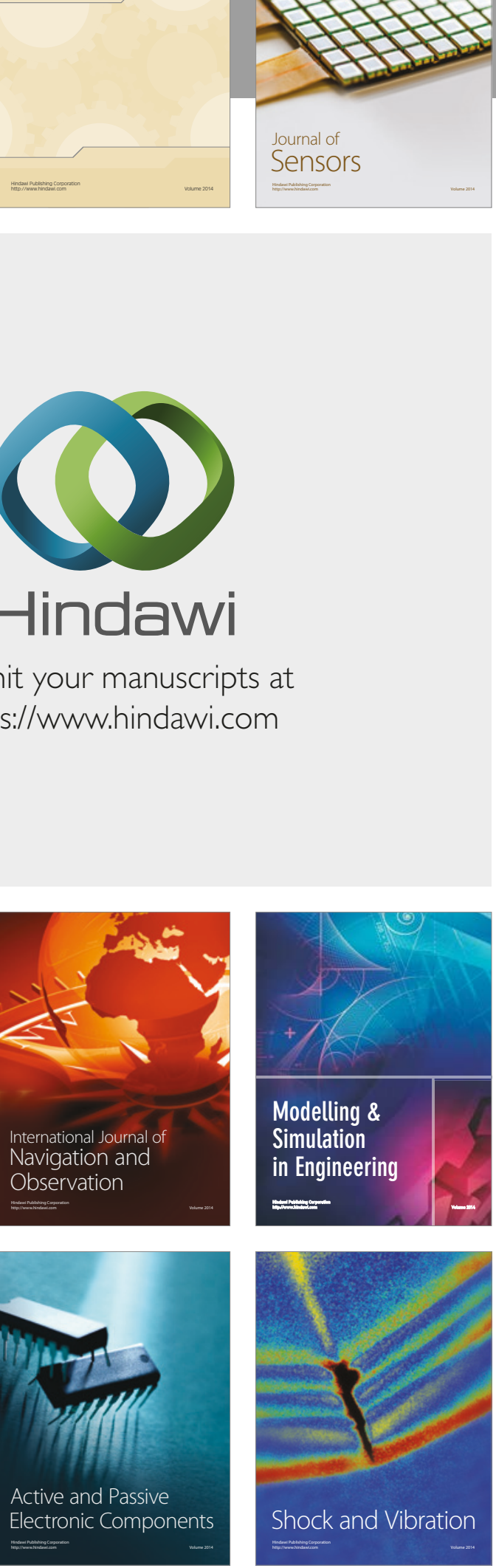
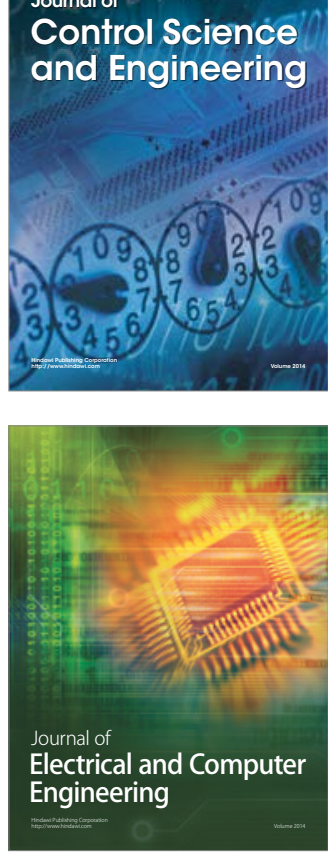

Distributed

Journal of

Control Science

and Engineering
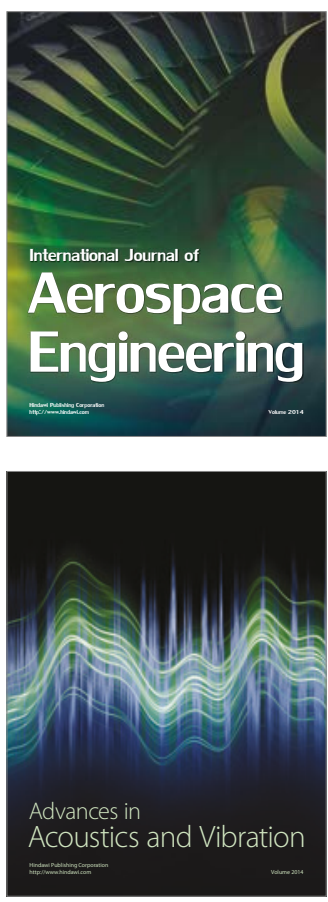

Sensor Networks 